\title{
COMMUTATIVITY OF RINGS WITH ABELIAN OR SOLVABLE UNITS
}

\author{
W. K. NICHOLSON ${ }^{1}$ AND H. J. SPRINGER
}

\begin{abstract}
A ring is called left suitable if idempotents can be lifted modulo every left ideal. These rings include all regular and all semiperfect rings. A left suitable ring with abelian group of units is commutative if it is either semiprime or 2-torsion-free. A left suitable ring with zero Jacobson radical and solvable group of units is commutative if it is 6-torsion-free.
\end{abstract}

Introduction. Lanski has shown [3] that a (von Neumann) regular ring is commutative if it is 6-torsion-free and has a solvable group of units. It follows from [4] that a semiperfect ring with abelian group of units is commutative if it is either semiprime or 2-torsion-free. The present paper extends these theorems to a class of "left suitable" rings. These rings are defined in $\S 1$ and the two theorems are proved in $\$ \S 2$ and 3.

Throughout the paper every ring will have an identity. The group of units and the Jacobson radical of a ring $R$ will be denoted by $R^{*}$ and $J(R)$ respectively. A ring $R$ is semiperfect if $R / J(R)$ is artinian and idempotents can be lifted modulo $J(R)$. If $R / J(R)$ is a division ring $R$ will be called local. A ring $R$ is (von Neumann) regular if given $a \in R$ there exists $b \in R$ with $a b a=a$. A ring $R$ is $n$-torsion-free if $n a=0$ with $a \in R$ implies $a=0$. Finally, $R$ is semiprime if $L^{2}=0, L$ a one-sided ideal of $R$, implies $L=0$.

1. Left suitable rings. The notion of lifting idempotents modulo an ideal is important in ring theory. The requirement that this be possible modulo any left ideal leads to an interesting class of rings.

1.1. Definition. If $L$ is a left ideal of a ring $R$ we say that idempotents can be lifted modulo $L$ if, given $x \in R$ such that $x^{2}-x \in L$, there exists $e^{2}=e \in R$ such that $e-x \in L$. A ring $R$ is called LEFT SUITABLE if idempotents can be lifted modulo every left ideal of $R$.

The following characterization of left suitable rings will be referred to several times.

1.2. Lemma. $A$ ring $R$ is left suitable if and only if for each $x \in R$ there exists $e^{2}=e \in R x$ such that $1-e \in R(1-x)$.

Proof. If $R$ is left suitable and $x \in R$, choose $e^{2}=e$ such that $e-x$

Received by the editors April 29, 1975.

AMS (MOS) subject classifications (1970). Primary 16A70; Secondary 16A30, 16 A32.

Key words and phrases. Group of units, commutativity, regular rings, semiperfect rings, lifting idempotents.

${ }^{1}$ The research for this author was supported by N.R.C. (Canada) grant number A8075. 
$\in R\left(x^{2}-x\right)$. Then $e \in R x$ and $1-e=(1-x)-(e-x) \in R(1-x)$. Conversely, if $e^{2}=e \in R x$ and $1-e \in R(1-x)$ then $e-x=e(1-x)$ $-(1-e) x \in R\left(x^{2}-x\right)$.

The class of left suitable rings is quite large. In particular, it contains all regular and all semiperfect rings. Indeed:

1.3. Proposition. If $R$ is a ring such that $R / J(R)$ is regular and idempotents can be lifted modulo $J(R)$ then $R$ is left suitable.

Proof. Let $x \in R$ and choose $y \in R$ such that $x-x y x \in J(R)$. Then $(y x)^{2}-y x \in J(R)$, so let $f^{2}=f$ be such that $f-y x \in J(R)$. We may assume $f \in R x$ since, if $u=1-f+y x$, then $f_{1}=u^{-1} f u$ satisfies $f_{1}^{2}=f_{1}$ $\in R x$ and $f-f_{1} \in J(R)$.

Now $x(1-f) \in J(R)$ so that $(1-f) x(1-f) \in J[(1-f) R(1-f)]$. Hence there exists $v \in R$ such that $(1-f) v(1-f)(1-x)(1-f)=1-f$. Put $g=(1-f) v(1-f)(1-x)$. Then $g^{2}=g \in R(1-x)$ and, since

$$
g(1-f)=1-f, \quad 1-g=f-g f \in R x .
$$

Hence we are finished by Lemma 1.2 with $e=1-g$.

The following example shows that the rings in Proposition 1.3 do not exhaust the class of left suitable rings.

1.4. Example. Let $F$ denote the rational numbers and let $L$ be the local subring of all rationals with odd denominators. If

$$
R=\left\{\left(q_{1}, q_{2}, \ldots, q_{n}, q, q, \ldots\right) \mid q_{i} \in F, q \in L, n \geqslant 1\right\}
$$

then, with component-wise operations, $R$ is a commutative ring with $J(R)=0$ and $R$ is not regular ( $L$ is a homomorphic image). However, the fact that $F$ and $L$ are both left suitable shows easily that the same is true of $R$.

The following property of left suitable rings will be referred to in both the remaining sections.

1.5. Proposition. Let $R$ be a left suitable ring in which every idempotent is central. Then every element in $R$ is the sum of a unit and an idempotent.

Proof. Given $x \in R$, let $e^{2}=e=a x$ and $1-e=b(1-x)$ where $a, b$ $\in R$. We may assume that $e a=a$ so that $a x a=a$ and $x a$ is an idempotent . But then

$$
x a=x(a x) a=x a(a x)=(x a) a x=a(x a) x=a x .
$$

Similarly we may assume $b(1-x) b=b$ and $(1-x) b=b(1-x)$. Since $e b=0=(1-e) a$, it follows that $x-(1-e)$ has inverse $(a-b)$.

2. Abelian units. If $R$ is a semiperfect ring and $R^{*}$ is abelian it follows from [4] that $R$ is commutative if it is either 2 -torsion-free or semiprime. In this section this result is extended to left suitable rings. The following result will be needed.

2.1. Lemma. Let $R$ be a ring for which $R^{*}$ is abelian. If either $R$ is 2-torsionfree or $R$ is semiprime then every idempotent in $R$ is central.

Proof. Let $e^{2}=e \in R$, let $r \in R$ and write $a=e r-e r e$. It suffices to 
show $a=0$ since an analogous argument shows $r e=e r e$. If $R$ is 2-torsionfree observe that $1-2 e$ and $1-a$ both lie in $R^{*}$ and so $(2 e) a=a(2 e)$. This means $2 a=2(e a)=2 a e=0$ so $a=0$. If $R$ is semiprime choose $s \in R$ and write $b=s e-e s e$. Then $1+a$ and $1+b$ lie in $R^{*}$ so $a b=b a$. Hence $a b=(e a) b=e b a=0$. But $b a=0$ means $s(e r-e r e)=e(s e r)-e(s e r) e$ and it follows that $L=\{e x-e x e \mid x \in R\}$ is a left ideal of $R$. Since $L^{2}=0$ it follows again that $a=0$.

The commutativity theorem now follows easily.

2.2. TheOREM. Let $R$ be a left suitable ring such that $R^{*}$ is abelian. Then $R$ is commutative if it is either semiprime or 2-torsion-free.

Proof. Lemma 2.1 shows that every idempotent in $R$ is central. The result then follows from Proposition 1.5.

The ring $R$ of upper triangular matrices over $G F(2)$ shows that the theorem is not true if $R$ is neither semiprime nor 2-torsion-free.

3. Solvable units. It was shown by Lanski [3, Theorem 12] that a 6-torsionfree regular ring $R$ is commutative provided $R^{*}$ is solvable. This section is devoted to generalizing this result to left suitable rings. The proof will appeal to the following result of Lanski [3, Lemma 5], [2, Theorem 9].

3.1. Proposition (LANSKI). Let $R$ be a semiprime ring which is 6-torsion-free. If $R^{*}$ is solvable then every idempotent in $R$ is central.

It is well known that a ring $R$ is local if and only if $x \in R^{*}$ or $1-x \in R^{*}$ for every $x \in R$. The following represents a weakening of this condition and will be needed below.

3.2. Proposition. Let $R$ be a ring and suppose $G \subseteq R^{*}$ is a group satisfying the following conditions:

(1) $x \in R$ implies $x \in G$ or $1-x \in G$,

(2) $x \in G$ implies $-x \in G$.

Then $G=R^{*}$ and $R$ is local.

Proof. Write $A=R-G$. It suffices to show that $A$ is an ideal of $R$.

Lemma. $A=\{a \in R \mid a+g \in G$ for all $g \in G\}$.

Proof. Suppose $a \in A$ and let $g \in G$ be arbitrary. Then $a \notin G$ so $g^{-1} a \notin G$ and consequently $1-g^{-1} a \in G$. This means $g-a \in G$ and so, replacing $g$ by $-g$, it follows that $a+g \in G$. Conversely, if $a+g \in G$ for all $g \in G$ and $a \notin A$ then a contradiction is achieved with $g=-a$.

Now we can show that $A$ is an ideal. Let $a, b \in A$ and let $g \in G$. Then $a-g \in G$ so $-a+g \in G$ and consequently $b-a+g \in G$. This shows $A$ is an additive subgroup. Next, $a b+g=a(b+g)+(1-a) g$ is in $G$ since $(1-a) g \in G$ and $a(b+g) \notin G$. Hence $A$ is a subring. Finally, if $x \notin A$ then $x \in G$ and so $x a \notin G$ and $a x \notin G$ (since $a \notin G$ ). This completes the proof of Proposition 3.2.

Incidentally, if $G \subseteq R^{*}$ satisfies only condition (1) $R$ is local and $G$ has index 1 or 2 in $R^{*}$. Indeed, if we denote $-G=\{-g \mid g \in G\}$, then the group $G_{1}=G \cup(-G)$ satisfies both conditions (1) and (2). 
3.3. TheOREM. Let $R$ be a left suitable ring such that $J(R)=0$ and $R$ is 6torsion-free. If, in addition, $R^{*}$ is solvable then $R$ is commutative.

Proof. Let $P$ denote a primitive ideal of $R$. Since $J(R)=0$ it suffices to show that $R / P$ is commutative. If $e^{2}=e \in R$ then $e R(1-e)=0$ by Proposition 3.1 and so, since $P$ is prime, either $e \in P$ or $(1-e) \in P$. Now let $\varphi: R \rightarrow R / P$ be the natural map and put $G=\left(R^{*}\right) \varphi$. If $x \varphi \in R / P$ write $x=e+u, e^{2}=e, u \in R^{*}$. Since either $e \in P$ or $(1-e) \in P$ it follows that either $x \varphi=u \varphi \in G$ or $1-x \varphi=(-u) \varphi \in G$. Thus $G$ satisfies the conditions of Proposition 3.2 and it follows that $R / P$ is local and $(R / P)^{*}=\left(R^{*}\right) \varphi$. Thus $R / P$ is a division ring ( $P$ is primitive) with a solvable group of units and so is commutative by a result of Hua [1]. This completes the proof.

The rings of $2 \times 2$ matrices over $G F(2)$ and $G F(3)$ show that the hypothesis of 6-torsion-free in Theorem 3.3 cannot be weakened to 2-torsion-free or 3torsion-free.

\section{REFERENCES}

1. Loo-Keng Hua, On the multiplicative group of a field, Acad. Sinica Science Record 3 (1950), 1-6. MR 12, 584.

2. C. Lanski, Subgroups and conjugates in semi-prime rings, Math. Ann. 192 (1971), 313-327. MR 44 \# 4047.

3. - Some remarks on rings with solvable units, Ring Theory, (Proc. Conf., Park City, Utah, 1971), Academic Press, New York, 1972, pp. 235-240. MR 49 \# 7311.

4. W. K. Nicholson, Semiperfect rings with abelian group of units, Pacific J. Math. 49 (1973), 191-198. MR 48 \#11185.

Department of Mathematics, University of Calgary, Calgary, Alberta T2N 1N4, CANADA 